\title{
ПРИМЕНЕНИЕ СИСТЕМ МЕЖОСТИСТОЙ ДИНАМИЧЕСКОЙ СТАБИЛИЗАЦИИ У ПАЦИЕНТОВ СО СТЕНОЗОМ ПОЯСНИЧНОГО ОТДЕЛА ПОЗВОНОЧНИКА НА ФОНЕ СНИЖЕНИЯ КОСТНОЙ ПЛОТНОСТИ
}

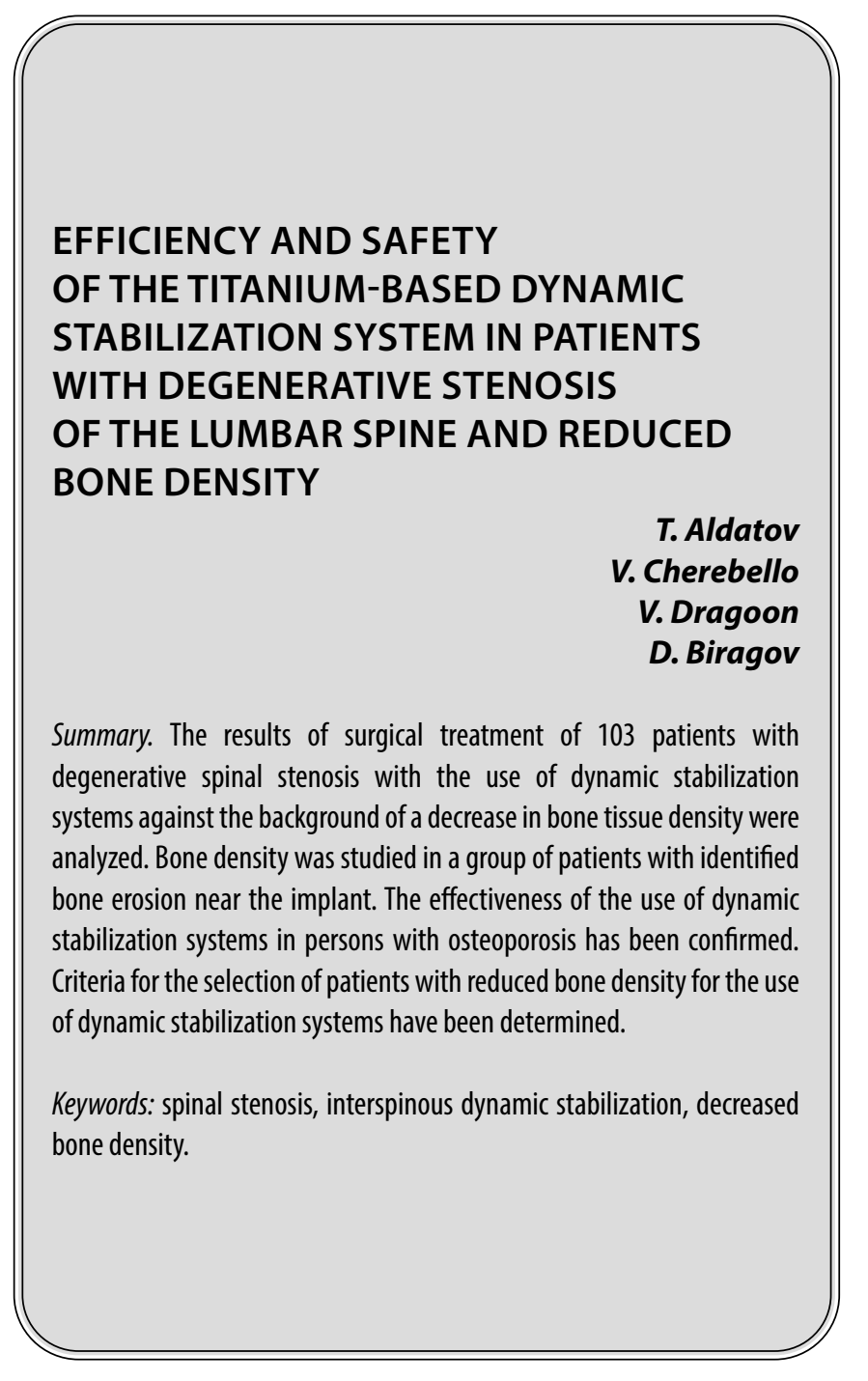

\section{Введение}

Д егенеративный стеноз поясничного отдела позвоночника является одним из самых распространенных заболеваний у лиц пожилого возраста и наиболее частой причиной перемежающейся хромоты [1,2]. Основными симптомами поясничного стеноза являются хронические боли в пояснице и нижних конечностях [3]. Данное заболевание серьезно ограничивает подвижность позвоночника, что в со-
Алдатов Тимур Сергеевич

Аспирант, Первый Санкт-Петербургский государственный медицинский университет им. Акад. И.П. Павлова, г. Санкт-Петербург aldatov.t@mail.ru

Черебилло Владислав Юрьевич

Д.м.н., профессор, Первый Санкт-Петербургский государственный медицинский университет им. Акад. И.П. Павлова, г. Санкт-Петербург cherebillo@mail.ru

Драгун Вадим Михайлович

К.м.н., дочент, Северо-Западный Государственный Медицинский Университет им И.И. Мечникова; главный нейрохирург ленинградской области, заместитель главного врача по хирургии Ленинградской областной клинической больницы dragun_vm@mail.ru

Бирагов Давид вячеславович Врач-нейрохирург, Медицинский чентр СОГАЗ,

г. Геленджик

davidsbir@yandex.ru

Аннотация. Проанализированы результаты хирургического лечения 103 пациентов с дегенеративным стенозом позвоночного канала с применением систем динамической стабилизации на фоне снижения плотности костной ткани. Исследована костная плотность у группы пациентов с выявленной костной эрозией вблизи импланта. Подтверждена эффективность применения систем динамической стабилизации у лиц с остеопорозом. Определены критерии отбора пациентов со сниженной костной плотностью для использования систем динамической стабилизации.

Ключевые слова: стеноз позвоночного канала, межостистая динамическая стабилизация, снижение плотности кости.

вокупности с болями в спине и нижних конечностях снижают активность и изменяют нормальную работу и повседневную жизнедеятельность пациента [4]. Консервативные методы лечения, такие как физиотерапия и назначение нестероидных противовоспалительных препаратов не эффективны для устранения симптомов поясничного стеноза [5]. Преимущество хирургической декомпрессии по сравнению с консервативными методами лечения в настоящее время является доказанным и подтверждено множеством клинических исследова- 
Таблица 1. Распределение пациентов по уровням поражения.

\begin{tabular}{|l|l|l|}
\hline $\begin{array}{l}\text { Уровень пораженного } \\
\text { сегмента }\end{array}$ & Количество пациентов в группе A & Количество пациентов в группе B \\
\hline L2/L3 & 3 & 2 \\
\hline$L 3 / L 4$ & 13 & 10 \\
\hline$L 4 / L 5$ & 22 & 25 \\
\hline$L 5 / S 1$ & 15 & 14 \\
\hline
\end{tabular}

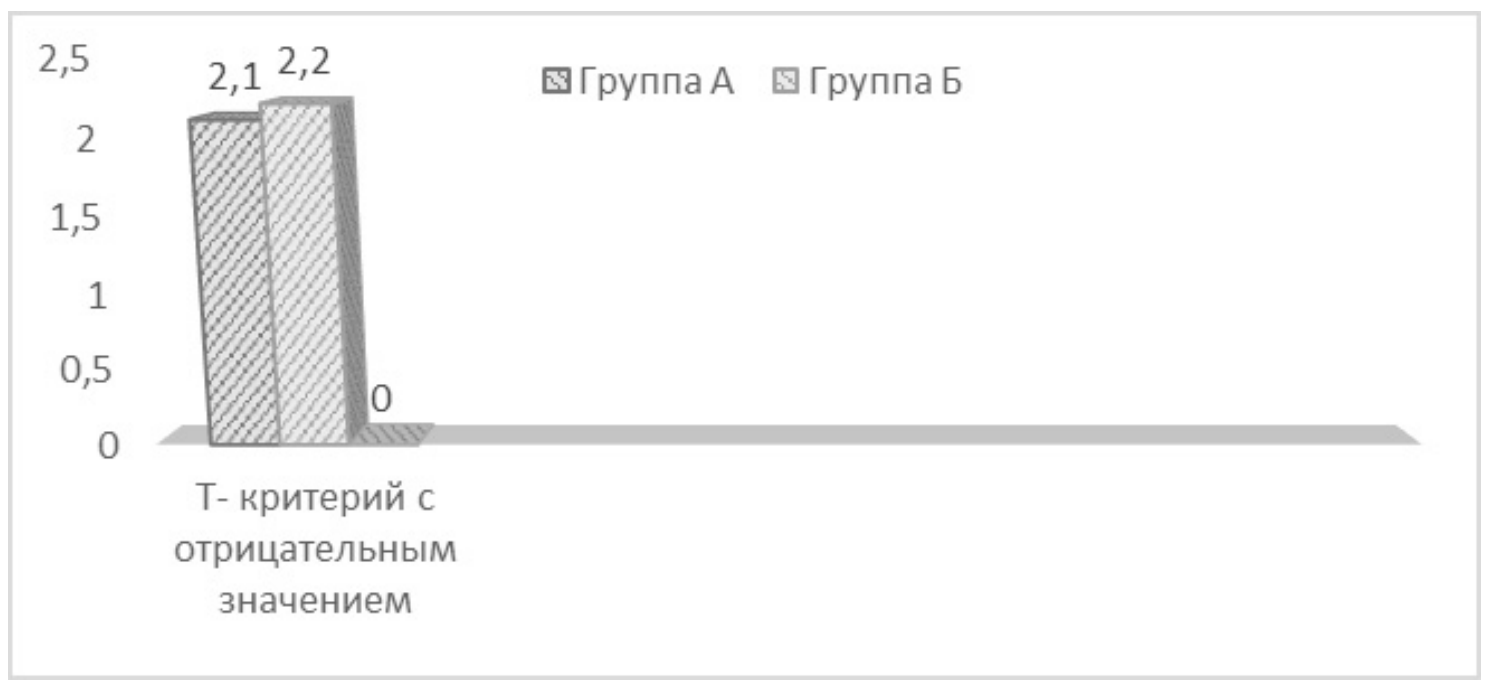

Диаграмма 1. Среднее значение минеральной плотности кости по Т- критерию с отрицательным значением.

ний [6]. Тем не менее, одна только декомпрессия не способна полностью устранить патологическое состояние позвоночника и может стать причинной будущей нестабильности в оперированном сегменте. [7]. Для уменьшения потенциального риска будущей нестабильности и деформации поясничного отдела позвоночника в последнее время все чаще прибегают к заднебоковому спондилодезу [8]. Спондилодез поддерживает объем центрального канала и фораминальную высоту, которые достигаются декомпрессией стенозированного сегмента позвоночника. Инструментальный спондилодез используется в качестве показанного и рекомендованного метода лечения при нестабильности позвоночника, либо в случаях, когда декомпрессия может привести к нестабильности позвоночника. Однако в виду того, что спондилодез приводит к нарушению движения в сросшихся сегментах, в некоторых исследованиях сообщалось об осложнениях, вызванных операцией таких как спондилоартроз и дегенерацию соседних уровней [9]. Стабилизация позвоночника после декомпрессии с помощью менее инвазивных, сохраняющих движения имплантов таких как Coflex и Diam обеспечивают третий вариант лечения, являющийся промежуточным между декомпрессией и спондилодезом [10].

\section{Материалы \\ и мето $\Delta$}

В проспективное двойное открыто контролируемое рандомизированное исследование была включена информация о 104 пациентах прооперированный с 2017 по 2019 годы в Ленинградской областной клинической больнице, ФГБУ «НМИЦ им. В.А. Алмазова» Минздрава России и на базе многопрофильного медицинского центра СОГАЗ Геленджик.

Все пациенты в исследовании имели анамнез заболевания более 6 месяцев. Основными клиническими проявлениями на момент хирургического лечения выступали боль в спине и нижних конечностях, компрессионно-ишемическая радикулопатия, нарушения двигательной активности. У всех пациентов диагноз Спинальный стеноз подтвержден инструментально при помощи МРТ пояснично- крестцового отдела позвоночника. Снижение плотности костной ткани подтверждено данными денситометрии поясничного отдела позвоночника. Среднее снижение минеральной плотности кости по Т- критерию представлено в диаграмме № 1. 
По половой принадлежности пациенты имели следующее распределение; мужчины 49 (47.1\%), женщины 55 (52.9\%). Распределение пациентов по уровням поражения представлено в таблице 1. В зависимости от установки того или иного импланта пациенты разделены на следующие 2 группы: первую группу (группа А) составили пациенты, которым выполнялась микрохирургическая декомпрессия позвоночного канала на поясничном уровне с установкой межостистой динамической системы Coflex (N = 53); вторую группу (группа Б) составили пациенты, которым также выполнялась микрохирургическая декомпрессия позвоночного канала на поясничном уровне, но с установкой межостистой динамической системы DIAM (N = 51). Средний срок наблюдения пациентов после операции составил 14.1 месяца (12-18 месяцев). Средний возраст пациентов составил 59,34 лет (50-85 лет).

Критерии включения пациентов в исследование

- Возраст пациентов старше 50 лет и наличие снижение плотности кости по данным денситометрии (от -1,2 SD до - 2,9 SD)

- Клинические признаки дегенеративного стеноза позвоночного канала на поясничном уровне, подтвержденные данными МРТ пояснично- крестцового отдела позвоночника.

- Отсутствие в анамнезе предшествующих операций на данном сегменте позвоночника.

Критерии исключения пациентов из исследования

- Пациенты с тяжелым остеопорозом.

- Пациенты с травматическими повреждениями позвоночника.

- Пациенты со спондилолистезом 2 и более степени.

- Пациенты с наличием противопоказаний к установке межостистого импланта.

\section{КАинические критерии оченки} полученных результатов

В результате исследования оценивалась динамика неврологического статуса, интенсивность и длительность болевого синдрома, дальность ходьбы до наступления болей. Оценка степени нарушений активности пациентов оценивалась нами по индексу Освестри (ODI). Освестри - широко распространенная шкала, применяемая для оценки выраженности нарушений жизнедеятельности, связанных с патологией позвоночника. Для оценки выраженности боли в поясничной области применялась визуальная аналоговая шкала (ВАШ). Также применялся опросник по боли раin DETECT. Опросник удобен для заполнения, так-как распределения болевых расстройств представлено в виде картинок, и объединено с опросником, обращенным на выявление признаком нейропатической боли.
Для статистической обработки материала применены пакеты прикладных программ IBM SPSS Statistica и Microsoft Office Excel 2016. Для оценки количественных данных использовались следующие критерии: t- критерий Стьюдента для независимых выборок, t- критерий Стьюдента для парных выборок, критерий Манна-Уитни. Для оценки качественных данных использовался критерий Хи- квадрат Пирсона. Для оценки нормальности распределения использовался критерий КолмогороваСмирнова. Степень пороговой статистической значимости при сравнении качественных и количественных признаков в исследуемых группах принимали равными 0,05 ( $\mathrm{P}<0,05)$. Каждый пациент, участвующий в исследовании, дал информированное согласие на участие в исследовании. Исследование проводилось с одобрения локального этического комитета Первого Санкт-Петербургского государственного медицинского университета им. акад. И.П. Павлова.

\section{Результаты}

В группе с установкой динамической системы Coflex средний возраст пациентов был несущественно ниже: $58,5 \pm 6,1$ лет, против 60,2 \pm 6,2 в группе DIAM. Подавляющее число пациентов обеих групп (47 пациента первой группы (87\%), и 44 пациента второй группы (88\%)) прооперированы на одном уровне. Наиболее часто производилось хирургическое лечение в сегменте L4-L5. (табл. 1). Показания к хирургическому лечению выставлялись на основании жалоб, анамнестических данных, данных объективного обследования, оценки неврологического дефицита и сопоставлении всего вышеперечисленного с данными инструментальных методов исследования.

Основными клиническими проявлениями заболевания у пациентов на дооперационном периоде были; люмбалгия (62\% больных), корешковые боли (75\% пациентов), симптомы нейрогенной перемежающей хромоты (54,5\%) пациентов. В подавляющем большинстве случаев (85\%) у пациентов отмечалось расстройство чувствительности в виде гипо- и анестезии в зоне иннервируемом пораженным корешком. У $69 \%$ пациентов расстройство чувствительности сочеталось со снижением рефлексов или слабостью в мышцах нижних конечностей.

Рентгенологически оценивались угол сегментарного лордоза на пораженном уровне, а также угол поясничного лордоза. Средний угол сегментарного лордоза

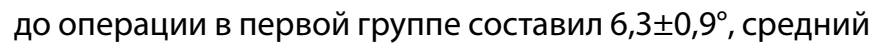
угол поясничного лордоза составил $45,4 \pm 3,4^{\circ}$. Средний угол сегментарного лордоза во второй группе до операции составил $6,1 \pm 1,1^{\circ}$, средний угол поясничного

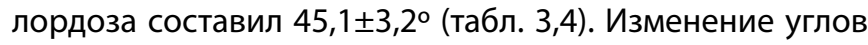
сегментарного и поясничного лордоза, оцениваемые 
Таблица 2. Оценка результатов хирургического лечения.

\begin{tabular}{|c|c|c|c|c|c|c|}
\hline Имплант & Coflex & & & Diam & & \\
\hline Срок наблюдения & До операции & $\begin{array}{l}6 \text { месяцев } \\
\text { после операции }\end{array}$ & $\begin{array}{l}12 \text { месяцев } \\
\text { после операции }\end{array}$ & До операции & $\begin{array}{l}6 \text { месяцев } \\
\text { после операции }\end{array}$ & $\begin{array}{l}12 \\
\text { месяцев после } \\
\text { операции }\end{array}$ \\
\hline $\begin{array}{l}\text { Оценка по индексу } \\
\text { Освестри }\end{array}$ & $59,2 \pm 12,7$ & $26,1 \pm 10$ & $23,1 \pm 8,3$ & $58,6 \pm 11,3$ & $24,6 \pm 9$ & $19,9 \pm 7,8$ \\
\hline Оценка по раin DETECT & $38,4 \pm 7,4$ & $25,1 \pm 7,8$ & $20,8 \pm 7$ & $39,8 \pm 7,2$ & $24,2 \pm 5,7$ & $20,4 \pm 6,4$ \\
\hline $\begin{array}{l}\text { Средняя фораминальная } \\
\text { высота в мм. }\end{array}$ & $14,0 \pm 2,7$ & $18,1 \pm 2,3$ & $17,2 \pm 2,1$ & $14,1 \pm 2,9$ & $17,7 \pm 1,7$ & $17,1 \pm 1,4$ \\
\hline $\begin{array}{l}\text { Средняя высота на уровне } \\
\text { заднего края диска в мм. }\end{array}$ & $5,4 \pm 1,7$ & $8,1 \pm 1,7$ & $7,5 \pm 1,6$ & $5,3 \pm 1,6$ & $6,4 \pm 1,5$ & $6,2 \pm 1,4$ \\
\hline Оценка по ВАШ & $7,1 \pm 1,6$ & $4,1 \pm 1,1$ & $3,4 \pm 1,2$ & $7,2 \pm 1,4$ & $3,5 \pm 1,3$ & $2,8 \pm 1,1$ \\
\hline
\end{tabular}

Таблица 3. Оценка динамики поясничного и сегментарного лордоза при установке импланта Coflex.

\begin{tabular}{|l|l|l|l|}
\hline Оцениваемый параметр & До операции & После операции & 12 месяцев после операции \\
\hline Сегментарный лордоз & $6,3 \pm 0,9$ & $5,5 \pm 1,0$ & $5,9 \pm 1,0$ \\
\hline Поясничный лордоз & $45,4 \pm 3,4$ & $43,4 \pm 2,9$ & $44,6 \pm 3,3$ \\
\hline
\end{tabular}

Таблица 4. Оценка динамики поясничного и сегментарного лордоза при установке импланта Diam.

\begin{tabular}{|l|l|l|l|}
\hline Оцениваемый параметр & До операции & После операции & $\begin{array}{l}12 \text { месяцев после } \\
\text { операции }\end{array}$ \\
\hline Сегментарный лордоз & $6,1 \pm 1,1$ & $5,8 \pm 1,2$ & $5,9 \pm 1,1$ \\
\hline Поясничный лордоз & $45,1 \pm 3,2$ & $43,9 \pm 3,4$ & $44,7 \pm 3,3$ \\
\hline
\end{tabular}

на 3-7 сутки, и через 12 месяцев после операции у первой группы пациентов представлены в таблице 3.

При проведении рентгенологических исследований через 12 месяцев после операции деформация во фронтальной плоскости определялась у одного пациента 1 группы. Сегментарная нестабильность оценивалась согласно критериям White A. A., Panjabi M.M. [11]. В первой группе сегментарная нестабильность выявлена у 2 пациентов (3,8\% пациентов). Во второй группе сегментарная нестабильность выявлена у 1 пациента (2\% пациентов) Интенсивность болевого синдрома до операции оценивалась по индексу Освестри, опроснику по боли pain DETECT, и визуальной аналоговой шкале. Обе группы показали значительное клиническое улучшение баллов по вышеуказанным опросникам. До хирургического лечения статистически значимых различий между пациентами в группах не отмечалось (табл. 2). Так же не отмечалось существенных различий при оценке фораминальной высоты и высоты заднего края диска в предоперационных снимках. В послеоперационном периоде выявлена отчетливая положительная динамика в виде увеличение вышеуказанных показателей в обеих группах. (табл. 2).

Результаты хирургического лечения представлены в таблицах 2,3,4.

Развитие эрозии вокруг импланта оценивалось по данным рентгенографии через 12 месяцев после операции.

\section{Осложнения}

Осложнения в группе А.Инфекционное осложнение наблюдалось у одного пациента с межостистым имплантом Coflex, в связи с чем, имплант был удален (1,9\%). Четырем пациентам выполнена повторная операция по поводу рецидива поясничного стеноза (7,5\%). У двух пациентов при попытке установки импланта, произошел перелом остистого отростка, данные пациенты из исследования исключены. У двух пациентов отмечались переломы остистого отростка в послеоперационном периоде (3,8\%). У одного пациента во время установки произошел 
перелом импланта (1,9\%). У шести пациентов отмечалась клинически незначимая миграция конструкции, не потребовавшая хирургической коррекции (11,3\%).

Осложнения в группе Б. Трем пациентам выполнена повторная операция по поводу рецидива поясничного стеноза (5,7\%). У четырех пациентов при попытке установки импланта отмечался перелом остистых отростков, данные пациенты из исследования исключены. У одного пациента отмечались перелом остистого отростка в послеоперационном периоде (2\%). У четырех пациентов отмечалась клинически незначимая миграция конструкции, не потребовавшая хирургической коррекции (7,8\%). Один пациент оперирован по поводу значительной миграции импланта (2\%).

\section{ОбсужАения}

Дегенеративный стеноз поясничного отдела позвоночника может быть, вызван множеством этиологических факторов. Самыми частыми из них являются: уменьшение мышечного каркаса поясничного отдела позвоночника, гипертрофия связочного аппарата позвоночника, протрузия межпозвонкового диска, пролиферация суставных отростков, гиперостоз задней границы позвонка. Развитие стеноза позвоночного канала вызывает постоянную компрессию спинномозгового корешка, вызывая боль в пояснице и нижних конечностях, а также радикулопатию. Наиболее часто течение стеноза позвоночного канала имеет хроническое течение. В дебюте заболевая боль отличается малой интенсивностью и может облегчаться изменением положения тела, в связи с чем пациенты могут длительное время не обращаться за медицинской помощью [12].

Методы лечение стеноза поясничного отдела позвоночника можно разделить на консервативный и хирургический. Консервативное лечение включает в себя: физиотрерапию, ЛФК (комплекс упражнений, направленных на укрепление поясничного отдела позвоночника), медикаментозную терапию, ношение корсета (направлено на уменьшение нагрузки на поясничный отдел позвоночника [13]. При неэффективности консервативной терапии прибегают к хирургическому лечению. На сегодняшнее время общепризнанными считаются две методики хирургического лечения: декомпрессия, включающая в себя широкую ламинэктомию, и декомпрессия с заднебоковой стабилизацией [28(14)]. По данным зарубежных авторов декомпрессия плюс заднебоковой спондилодез является одной из самых частых операций при стенозе поясничного отдела позвоночника у пожилых людей [15]. Однако, по результатам исследований, исходная биомеханика позвоночника при этом виде операции нарушается. По данным, Юань и соавторов спондилодез существенно повышает объём движений в смежных сегментах по сравнению с дооперационным уровнем, что может стать причиной ускоренной дегенерации соседних уровней и ещё более усугубить боль в пояснице [16].

Применение систем межостистой динамической стабилизации не оказывает существенного влияния на подвижность соседних сегментов, а также способствует распределению нагрузки на поражённом сегменте, тем самым делая его подвижность аналогичной здоровой. Таким образом, обеспечивается стабильность и не происходит повреждения соседних сегментов [17]. Прошлые исследования показали, что применение систем межостистой динамической стабилизации позволяет восстановить высоту межпозвонкового промежутка в ближайшем послеоперационном периоде [18]. Однако при длительном наблюдении (от 1 до 4-х лет) в большинстве случаев высота межпозвонкового диска возвращалась на предоперационный уровень [19].

В нашем исследовании мы сравнивали фораминальную высоту и высоту на уровне диска при различных системах динамической стабилизации, а также выясняли, насколько системы динамической стабилизации способствуют эрозии остистых отростков у пациентов со сниженной костной плотностью. По данным Park и соавторов, костную эрозию следует рассматривать как осложнение установки импланта, так как костная эрозия может вызвать боль и дискомфорт в пояснице и это может снизить удовлетворение от хирургического лечения [19]. Впоследствии при прогрессирующей костной эрозии может произойти перелом остистого отростка, либо миграция межостистого импланта. По данным литературы, через 1 год после хирургического лечения эрозия вокруг импланта Coflex достигала 14\%, а при 4-х летнем периоде наблюдения 47\% [20].

Результаты наших исследований показали эффективность применения систем межостистой динамической стабилизации у пациентов с дегенеративным стенозом поясничного отдела позвоночника на фоне снижения костной плотности. Все пациенты в исследовании отмечали положительный результат по индексу Освестри (ODI), опроснику по боли раin DETECT и визуальной аналоговой шкале. Эрозия остистых отростков через 12 месяцев после операции наблюдалась у 11 пациентов группы А, что составляет 21\%. Таким образом эрозия вокруг импланта более выражена у пациентов со сниженной костной плотностью.

Наше исследования является новым по сравнению с предыдущими исследованиями, так как включает в себя оценку систем динамической стабилизации у пациентов на фоне снижения костной плотности. Последующие исследования должны быть направлены 
на дальнейшее наблюдение за пациентами и оценкой рентгенологических и клинических параметров, а также оценкой костной эрозии вокруг импланта.

\section{ВывO $\triangle \mathrm{bl}$}

Микрохирургическая декомпрессия с последующей межостистой динамической стабилизацией является методикой выбора у пациентов пожилого возраста ввиду минимальности послеоперационной раны, небольшой длительности операции и незначительности кровопоте- ри. Результаты данного исследования позволяют судить о эффективности применения систем межостистой динамической стабилизации таких как Coflex и DIAM у пациентов со сниженной костной плотностью.

Согласно нашему исследованию у данной категории пациентов предпочтительнее использовать имплант DIAM, ввиду отсутствия развития эрозии вокруг импланта, а также более значимого улучшения показателей по индексу Освестри и визуальной аналоговой шкале.

\section{ЛИТЕРАТУРА}

1. Fay LY, Wu JC, Tsai TY, Wu CL, Huang WC and Cheng H: Dynamic stabilization for degenerative spondylolisthesis: Evaluation of radiographic and clinical outcomes. Clin Neurol Neurosurg 2013;115;535-541.

2. Берснев В.П., Драгун В. М., Микаилов С. Ю., Кудзиев А. В. Хирургическое лечение поясничного спинального стеноза сприменением межостистых имплантирующих устройств // Политравма.— 2016. - № 1._- C. 18-23. [Bersnev VP, Dragun VM, Mikailov SYu, Kudziev AV. Surgical treatment of lumbar spinal stenosis using interspinous implant devices. Politrauma. 2016;(1):18-23.

3. Никитин А. С., Асратян С. А., Камчатнов П. Р. Стеноз поясничного отдела позвоночника // Журнал неврологии и психиатрии им. С. С. Корсакова. - 2015.T. 115. - № 7.- C. 130-140. [Nikitin AS, Asratyan SA, Kamchatnov PR. Stenosis of the lumbar spine. Journal of neurology and psychiatry C.C. Korsakova. 2015;115(7):130-140.

4. Choudhri TF, Mummaneni PV, Dhall SS, Eck JC, Groff MW, Ghogawala Z, Watters WC III, Dailey AT, Resnick DK, Sharan A, et al: Guideline update for the performance of fusion procedures for degenerative disease of the lumbar spine. Part 4: Radiographic assessment of fusion status. J Neurosurg Spine 2014;21;23-30.

5. R. A. Deyo, S. K. Mirza, B. I. Martin, et al., Trends, major medical complications, and charges associated with surgery for lumbar spinal stenosis in older adults, Jama $3032010 ; 13 ; 1259$.

6. Weinstein JN, Tosteson TD, Lurie JD, et al. Surgical versus nonoperative treatment for lumbar spinal stenosis four-year results of the Spine Patient Outcomes Research Trial. Spine (Phila Pa 1976). 2010;35(14);1329-1338.

7. Resnick DK, Watters WC III, Sharan A, et al. Guideline update for the performance of fusion procedures for degenerative disease of the lumbar spine. Part 9: lumbar fusion for stenosis with spondylolisthesis. J Neurosurg Spine. 2014;21(1);54-61.

8. H. W. Bae, S. S. Rajaee, L. E. Kanim, Nationwide trends in the surgical management of lumbar spinal stenosis, Spine 2013; 38 (11); 916.

9. M. Etminan, F. P. Girardi, S. N. Khan, et al., Revision strategies for lumbar pseudarthrosis, Orthop. Clin. N. Am. 2002;33 (2); 381.

10. T. J. Erica, J. R. Kamerlink, M. Quirno, et al., Survivorship of coflex interlaminar interspinous implant, SAS J. (2009);3 (2); 59.

11. White A. A., Panjabi M. M. Clinical Biomechanics of the Spine, 2nd ed. // Philadelphia: Lippincott. 1990. (2). C. 18-20.

12. Passmore SR, Johnson MG, Kriellaars DJ, PelleckV, Enright A and Glazebrook CM: Fitts's Law using lower extremity movement: Performance driven outcomes for degenerative lumbar spinal stenosis. Hum Mov Sci 2015;44; 277-286.

13. Yu SW, Yang SC, $\mathrm{Ma} \mathrm{CH}, \mathrm{Wu} \mathrm{CH}$, Yen CY and Tu YK: Comparison of Dynesys posterior stabilization and posterior lumbar interbody fusion for spinal stenosis $\mathrm{L} 4 \mathrm{~L} 5$. Acta. Orthop. Belg. 2012; 78;230-239.

14. Yang M, Li C, Chen Z, Bai Y and Li M: Short term outcome of posterior dynamic stabilization system in degenerative lumbar diseases. Indian J. Orthop. 48: 574-581, 2014.

15. Ai-min Li, Xiang Li, Zhong Yang. International Journal of Surgery 2017;40;60-67

16. W. Yuan, Q. Su, T. Liu, et al., Evaluation of Coflex interspinous stabilization following decompression compared with decompression and posterior lumbar interbody fusion for the treatment of lumbar degenerative disease: A minimum 5-year follow-up study, J. Clin. Neurosci. 2017;35; 24-29.

17. Park SC, Yoon SH, Hong YP, Kim KJ, Chung SK, Kim HJ. Minimum 2-year follow-up result of degenerative spinal stenosis treated with inter-spinous U (Coflex). J Korean Neurosurg Soc. 2009;46;292-299.

18. Celik H, Derincek A, Koksal I. Surgical treatment of the spinal stenosis with an interspinous distraction device: do we really restore the foraminal height? Turk Neurosurg. 2012;22;50-54.

19. Park P, Garton HJ, Gala VC, Hoff JT, McGillicuddy JE. Adjacent segment disease after lumbar or lumbosacral fusion: review of the literature. Spine (Phila Pa 1976). 2004;29;1938-1944.

20. Zang L, Du P, Hai Y, Su QJ, Lu SB, Liu T. Device related complications of the Coflex interspinous process implant for the lumbar spine. Chin Med J (Engl). 2013;126;2517-2522.

○ Алдатов Тимур Сергеевич ( aldatov.t@mail.ru), Черебилло Владислав Юрьевич ( cherebillo@mail.ru),

Драгун Вадим Михайлович (dragun_vm@mail.ru ), Бирагов Давид Вячеславович (davidsbir@yandex.ru ).

Журнал «Современная наука: актуальные проблемы теории и практики» 